\title{
Controlled and spontaneous magnetic field generation in a gun-driven spheromak
}

\author{
S. Woodruff, B. I. Cohen, E. B. Hooper, H. S. Mclean, B. W. Stallard, \\ D. N. Hill, and C. T. Holcomb \\ Lawrence Livermore National Laboratory, Livermore, California 94550 \\ C. Romero-Talamas \\ California Institute of Technology, Pasadena, California 91125 \\ R. D. Wood \\ Lawrence Livermore National Laboratory, Livermore, California 94550 \\ G. Cone and C. R. Sovinec \\ University of Wisconsin, Madison, Wisconsin 53706
}

(Received 11 October 2004; accepted 27 January 2005; published online 13 April 2005)

\begin{abstract}
In the Sustained Spheromak Physics Experiment, SSPX [E. B. Hooper, D. Pearlstein, and D. D. Ryutov, Nucl. Fusion 39, 863 (1999)], progress has been made in understanding the mechanisms that generate fields by helicity injection. SSPX injects helicity (linked magnetic flux) from $1 \mathrm{~m}$ diameter magnetized coaxial electrodes into a flux-conserving confinement region. Control of magnetic fluctuations $\left(\delta B / B \sim 1 \%\right.$ on the midplane edge) yields $T_{e}$ profiles peaked at $>200 \mathrm{eV}$. Trends indicate a limiting beta $\left(\beta_{e} \sim 4 \%-6 \%\right)$, and so we have been motivated to increase $T_{e}$ by operating with stronger magnetic field. Two new operating modes are observed to increase the magnetic field: (A) Operation with constant current and spontaneous gun voltage fluctuations. In this case, the gun is operated continuously at the threshold for ejection of plasma from the gun: stored magnetic energy of the spheromak increases gradually with $\delta B / B \sim 2 \%$ and large voltage fluctuations $(\delta V \sim 1 \mathrm{kV})$, giving a $50 \%$ increase in current amplification, $I_{\text {tor }} / I_{\text {gun }}$. (B) Operation with controlled current pulses. In this case, spheromak magnetic energy increases in a stepwise fashion by pulsing the gun, giving the highest magnetic fields observed for SSPX $(\sim 0.7 \mathrm{~T}$ along the geometric axis). By increasing the time between pulses, a quasisteady sustainment is produced (with periodic good confinement), comparing well with resistive magnetohydrodynamic simulations. In each case, the processes that transport the helicity into the spheromak are inductive and exhibit a scaling of field with current that exceeds those previously obtained. We use our newly found scaling to suggest how to achieve higher temperatures with a series of pulses. (C) 2005 American Institute of Physics. [DOI: 10.1063/1.1878772]
\end{abstract}

\section{INTRODUCTION}

SSPX (Sustained Spheromak Physics Experiment) was designed to explore the physics of confinement and magnetic field generation in a spheromak. Although magnetic field generation has been studied for some time, ${ }^{1}$ it remains a fruitful area of research. The original spheromak formation scheme is attributed to Alfvén, ${ }^{2}$ and Lindberg was the first to observe flux amplification to result from a magnetohydrodynamic (MHD) kink. ${ }^{3}$ By use of a magnetized coaxial gun, Turner et al. demonstrated fast formation $\left(\tau_{\text {form }} \sim \tau_{\text {Alfven }}\right){ }^{4}$ and Jarboe et al. showed slow formation. ${ }^{5}$ Later, spheromak magnetic fields were generated by induction. ${ }^{6}$ Recently, two spheromaks generated by two separate guns have been merged to form a single spheromak. ${ }^{7-9}$ The $n=1$ mode that results from a kink instability of the open flux was directly associated with current drive and field buildup before experiment, labeled the "dough-hook"10 and seen in threedimensional (3D) MHD simulations. ${ }^{11}$ Magnetic field generation processes in spheromaks are thought to be similar to those that form galactic magnetic fields ${ }^{12}$ and some evidence exists for the magnetohydrodynamic dynamo, ${ }^{13}$ thought to be an important process in the generation of astrophysical magnetic fields.

Confinement improvements in SSPX give peak $T_{e}$ $>200 \mathrm{eV}$ and core electron thermal diffusivities of $\chi_{e}$ $\sim 10 \mathrm{~m}^{2} / \mathrm{s}$ (previously $T_{e} \sim 120 \mathrm{eV}$ and $\chi_{e} \sim 50 \mathrm{~m}^{2} / \mathrm{s}$ were reported $^{14}$ ). There is evidence for island formation, indicative of toroidal surfaces, and most SSPX temperature data are bounded by a $\beta_{e} \sim 5 \% .{ }^{15}$ Given a limiting $\beta$ (not necessarily a fundamental $\beta$ limit), an increase of temperature is obtained by increasing the magnetic field strength $\left(T \sim \beta B^{2}\right)$, which motivates the work presented here.

In SSPX, formation with a wide variety of initial conditions gives rise to the same linear scaling of the edge poloidal magnetic field with the injected current observed as a $B_{p} \sim 0.0065 I_{\text {gun }}$ (Ref. 16) [much the same scaling as observed in SPHEX of $B_{p} \sim 0.0075 I_{\text {gun }}$ (Ref. 17)]. This scaling is produced during the period that the $n=1$ mode is present, caused by the dough-hook seen in all electrostatically driven spheromaks. Further, when a strong electrostatic driving field is used in MHD simulations of either gun geometries or simple cylinders, a strong current-driven $n=1$ instability ex- 


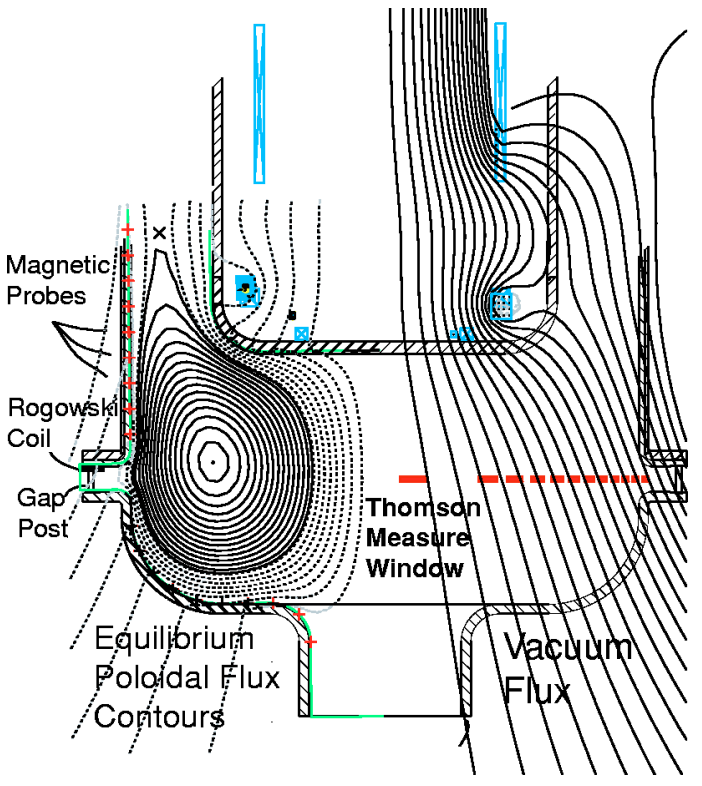

FIG. 1. SSPX and major components.

cited on open field lines of a plasma pinch configuration is generally seen. ${ }^{11,18}$ It has been found for these simulations that the peak field that can be obtained also scales as a function of the gun voltage and Lundquist number (hence, also the gun current).

Here we report two new methods of magnetic field generation with reference both to SSPX experimental data and results from resistive MHD simulations in both cylindrical and more SSPX-like geometries that exceed this linear scaling. While the emphasis has been to understand the processes by which helicity is injected into a spheromak, another equally important end is to enhance performance by pushing towards higher field strengths and higher temperatures. Towards this end an empirically derived scaling model ${ }^{19}$ is pre- sented that details means for accessing these interesting regimes.

The text is structured as follows. Section II entails a description of the experiment and of the simulation tools used here. Section III contains an analytic model based on the inductive nature of the gun impedance during helicity injection. $^{20}$ In Sec. IV, new results related to the two new operating modes are presented. Section V presents a discussion of the implications of these results for the scaling to a high temperature spheromak. Section VI contains the conclusions.

\section{EXPERIMENT AND SIMULATION TOOLS}

SSPX is a coaxial-gun-driven spheromak, similar in design to the version of the Compact Torus Experiment (CTX) (Ref. 21) that obtained a transient $400 \mathrm{eV}$ electron temperature in decay. SSPX is larger in volume by a factor of 8: it has a $1 \mathrm{~m}$ diameter gun of equal radius to the flux conserver (in CTX, the diameter was $40 \mathrm{~cm}$ ). Nine independently programmable field coils generate the vacuum field (see Fig 1 and Ref. 14). In this text, reference is made to a 10-point Profile Thomson Scattering (PTS) diagnostic, two-chord $\mathrm{CO}_{2}$ interferometer, fast charge-coupled device imaging camera, and edge magnetic probes. Base pressures as low as $10^{-9}$ Torr have been achieved by baking, helium shot conditioning, glow discharge cleaning, and Ti gettering, giving the burn-through of most impurities and low radiated power $\left(<P_{\text {gun }} / 10\right)$. The circuit that drives SSPX is shown in Fig. 2: a $0.5 \mathrm{MJ}$ inductance, resistance, and capacitance, LRC circuit for the formation pulse, and 1.5 MJ pulse-forming network that can deliver constant current pulse of $200 \mathrm{kA}$ for around $2 \mathrm{~ms}$. Typical operating parameters are shown in Table I. The circuit impedance exceeds that of the spheromak several fold, which means that the circuit acts as a current source. The voltage drop across the electrodes is measured with a voltage divider mounted on the gun.

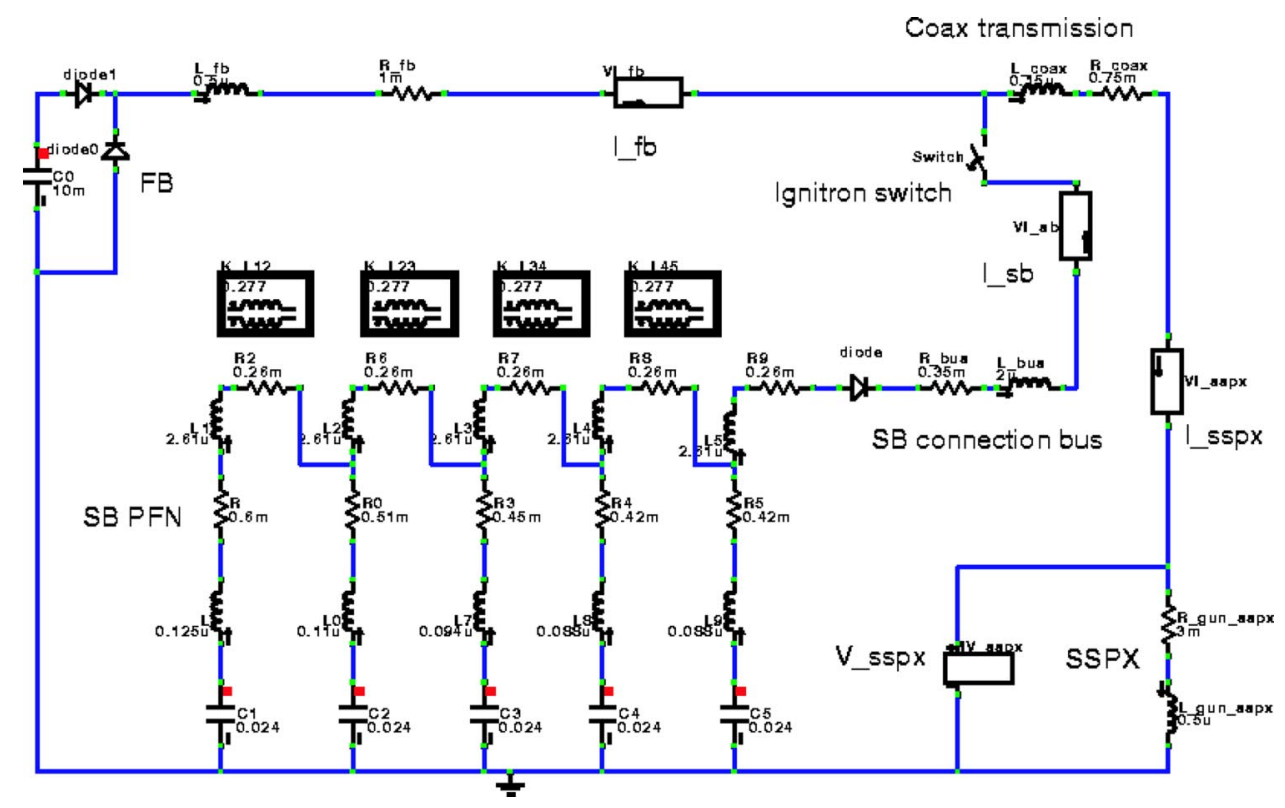

FIG. 2. SSPX gun circuit. 
TABLE I. Typical parameters for SSPX.

\begin{tabular}{lc}
\hline \hline Parameter & Value \\
\hline Major radius (m) & 0.5 \\
Minor radius (m) & 0.2 \\
Plasma current (kA) & 400 \\
Magnetic field $B_{0}(\mathrm{~T})$ & 0.5 \\
Pulse length (ms) & 3.5 \\
Density $\left(\mathrm{m}^{-3}\right)$ & $2-10$ \\
Electron temperature $(\mathrm{eV})$ & 200 \\
Ion temperature $(\mathrm{eV})$ & 600 \\
$\beta(\%)$ & 10 \\
\hline \hline
\end{tabular}

The NIMROD three-dimensional resistive-MHD $\operatorname{code}^{22,23}$ is used here as a primary tool for understanding magnetic field generation in the spheromak. The simulation domains are assumed to be bounded with perfect conductors so that magnetic field components normal to the boundaries and electric fields parallel to the boundaries vanish except for time-independent, applied background vacuum magnetic fields. The insulating gap required for applying dc voltage is modeled by specifying a tangential electric field along the outboard bounding wall in cylindrical-geometry computations and along the upstream end of the domain in gungeometry computations. SSPX simulations employed geometries with aspect ratios (vertical height over radius) in the range of 1-1.5 (SSPX is near unity in aspect ratio), major radius $0.5-1 \mathrm{~m}$ (SSPX has $0.5 \mathrm{~m}$ major radius), maximum magnetic fields $1-2 \mathrm{~T}$, plasma mass densities $10^{4}-10^{5}$ larger than in SSPX to reduce the disparity in Alfvén and resistive times, i.e., to artificially reduce the magnetic Lundquist number which is above $S \sim 10^{5}$ in hot SSPX discharges $\left(T_{e}\right.$ $>100 \mathrm{eV}$ ) to a more manageable $10^{3}<S<10^{4}$ in the simulations (where we use the major radius as the characteristic length scale in the definitions of the Alfvén and resistive times). In most of these simulations, fixed constants have been assumed for the values of the electrical resistivities and thermal conductivities.

Time histories of the global parameters in the experiment (such as spheromak helicity content and field energy) are inferred by fitting equilibria generated by the CORSICA (Ref. 24) code to the injected current and edge magnetic field measured in the poloidal plane. The current profile is characterized by the ratio of current to field, $\lambda=\mu_{0} \mathbf{J} \cdot \mathbf{B} / B^{2}$, as is customary in predominantly force-free plasmas. In the fits used here, the $\lambda$ profile is modeled inside the separatrix as $\lambda=\lambda_{\text {edge }}\left(1+\alpha \bar{\psi}^{n}\right) /(1+\alpha)$ where $\bar{\psi}$ is a normalized flux, varying from 0 on the magnetic axis to 1 on the separatrix, $\alpha$ is a fitting parameter, and $\lambda_{\text {edge }}=\lambda_{\text {gun }}=\lambda$ on the open field lines outside of the separatrix. $\lambda_{\text {gun }}=\mu_{0} I_{\text {gun }} / \psi_{\text {gun }}$, where $I_{\text {gun }}$ and $\psi_{\text {gun }}$ are the gun current and flux. The gun voltage is $V_{\text {gun }}$.

\section{HELICITY INJECTION BY INDUCTIVE PROCESSES}

Spheromak magnetic field generation is described with the use of "magnetic helicity." This is a measure of the linkage of the magnetic flux, is additive, and is conserved glo- bally in instances where magnetic energy is not e.g., in reconnections. ${ }^{20}$ The helicity-injection rate of the gun-driven spheromak is usually expressed in terms of the gun voltage and the flux linking two coaxial electrodes: $\dot{K}=2 V_{\text {gun }} \psi_{\text {gun }}$, and the spheromak global helicity evolution is expressed as

$$
\begin{aligned}
K(t)= & \exp \left(\int_{0}^{t} \frac{-d t}{\tau_{K}}\right) \\
& \times \int_{0}^{t} 2 V_{\text {gun }}\left(t^{\prime}\right) \psi_{\text {gun }}\left(t^{\prime}\right) \exp \left(\int_{0}^{t^{\prime}} \frac{d t^{\prime \prime}}{\tau_{K}}\right) d t^{\prime} .
\end{aligned}
$$

For time scales short compared with the helicity dissipation time, $\tau_{\mathrm{K}}\left(\mathrm{t} / \tau_{\mathrm{K}} \ll 1\right)$ the integral can be simplified to

$$
K(t)=\int_{0}^{t} 2 V_{\text {gun }}(t) \psi_{\text {gun }}(t) d t .
$$

In order to understand what it means to "inject helicity," it has been useful to determine which processes dominate $V_{\text {gun }}$ and to control these processes in the experiment. We have considered the following contributing factors to the gun voltage:

$$
\begin{aligned}
V_{\text {gun }}(t)= & V_{\text {sheath }}(t)+V_{\text {edge }}(t)+\frac{d}{d t}\left[L(t) I_{\text {gun }}(t)\right]+\delta V_{\text {gun }}(t) \\
& +\Delta V_{\text {gun }}(t),
\end{aligned}
$$

where $V_{\text {gun }}$ is that voltage measured at the gun, $V_{\text {sheath }}$ is a cathode sheath drop, $V_{\text {edge }}$ is the resistive drop in the cold edge plasma, and $\delta V_{\text {gun }}+\Delta V_{\text {gun }}$ are terms related to anomalous resistive processes. ${ }^{25}$ All of the terms except the $d / d t\left(L I_{\text {gun }}\right)$ inductive term are due to processes that dissipate helicity (sheaths, resistive edge, etc.). The thesis of this paper is that the main contribution to the useful helicity-injection rate for discrete, controllable events originates with the $d / d t\left(L I_{\text {gun }}\right)$ term, for which we can assume that the gun-flux injector flux $\psi_{\text {gun }}$ is invariant (see Discussion). We can define the effective helicity-injection rate in terms of the inductive plasma processes as follows:

$$
\begin{aligned}
& K_{e f f}(t)=\int_{0}^{t} 2 \dot{L}(t) I \psi_{\text {gun }}(t) d t+\int_{0}^{t} 2 \dot{I}(t) L \psi_{\text {gun }}(t) d t \\
& \frac{d K_{e f f}}{d t}=2 \dot{L} I \psi_{\text {gun }}+2 \dot{I} L \psi_{\text {gun }} .
\end{aligned}
$$

In Eq. (5) and below, $L$ and $\dot{L}$ are redefined as appropriate time averages, consistent with the experimental observation that in many experimental discharges one or the other is nearly constant for extended times.

To illustrate the inductive generation of helicity, the two main sources of impedance understood to dominate during helicity injection are shown in Fig. 3. For clarity, these are labeled type I processes and type II processes. The processes have analogs. Type I processes exhibit geometric changes like the plasma arcades of a Jacobs ladder: an area change results in a large $d L / d t$ term and so the first term on the right side of Eq. (5) is dominant. Type II processes occur like ramping current up in a fixed geometry, like a circuit induc- 


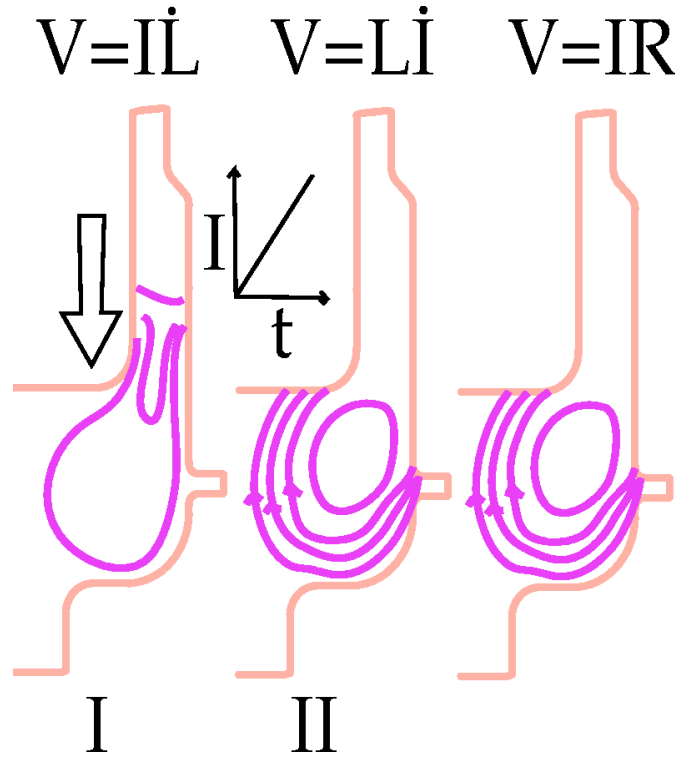

FIG. 3. Schematic of the processes that govern the gun voltage in SSPX.

tor which gives rise to a $d I / d t$ term, making the second term on the right side of Eq. (5) dominant. Therefore, to add helicity to a spheromak it is necessary to choose processes that have the characteristic of inductive impedance. Two processes that give an inductive impedance are described in the following analytic examples.

\section{A. Analytic expression of type I process}

Consider the instance that a single current sheet is expelled from the gun. If this occurs on a time scale that is short compared with the rate of change of gun current then $I_{\text {gun }} \sim$ const. By ignoring resistive impedance (assuming that this will be small in comparison with the inductive component) the gun voltage is $V_{\text {gun }}=I_{\text {gun }} \dot{L}$, and the effective helicity-injection rate is $\dot{K}_{\text {eff }}=2 \psi_{\text {gun }} I_{\text {gun }} \dot{L}$.

This injection rate can be rewritten in terms of the gun current alone by considering the following. In order for the sheet to be released from the gun, a pressure balance in the gun must be met in which the axial constraining force of the radial vacuum field is balanced by the $\mathbf{J} \times \mathbf{B}$ force acting on the current sheet. The only control parameter that is varied during this ejection process is the gun current, and so the pressure balance is often expressed in terms of a threshold current $I_{\text {thresh }}$. Equating magnetic pressures, the equality $B_{\theta}$ $=B_{p}$ is obtained for the expulsion of a current sheet. Expressing the gun flux in terms of the gun current by using a sharp boundary model (see Appendix B) one obtains an expression for the gun flux at the time of ejection in terms of the gun current and the gun geometry,

$$
\psi_{\text {gun }}=\mu_{0} I_{\text {thresh }} \Delta / 4,
$$

where the interelectrode gap width is $\Delta$ and the radius is $R$. Finally, the helicity-injection rate can be written in terms of the threshold current, geometry, and rate of change of inductance due to the length expansion of the current sheet:

$$
\dot{K}_{\text {eff }}=\mu_{0} I_{\text {thresh }}^{2} \dot{L} \Delta / 2 .
$$

The helicity content of the spheromak can be determined by the use of Eq. (7) and knowledge of the inductance of the current sheet expanding from the gun approximated as a coaxial cylinder: $L=\left(\mu_{0} / 2 \pi\right) l_{\text {sheet }} \ln \left(r_{2} / r_{1}\right)$, where $r_{2}$ and $r_{1}$ are the outer and inner electrode radii, respectively, $l_{\text {sheet }}$ is the length of the sheet (including both gun- and flux-conserver regions), giving $L \sim 100 \mathrm{nH}$. By combining the two expressions for $L$ and $\dot{K}$, a geometrical relationship for the magnetic helicity introduced with each current sheet is obtained. Integrating with respect to time, the helicity of the spheromak can be predicted from knowledge of the threshold current alone: $K=9 \times 10^{-15} I_{\text {thresh }}^{2} \mathrm{~Wb}^{2}$.

\section{B. Analytic expression of type II process}

The inductance of a screw pinch can be expressed by considering the limiting case where the column and return current form a coaxial section of fixed geometry (see Appen$\operatorname{dix} \mathrm{C}$ ). Before the onset of asymmetry and before a spheromak is established (for which $\psi_{\text {pol }} \sim \psi_{\text {tor }} \sim \psi_{\text {gun }}$, i.e., before flux amplification occurs), the injection rate can be stated as

$$
\dot{K}_{\text {eff }} \approx 2 L \psi_{\text {gun }} \dot{I}_{\text {gun }},
$$

where a simple model for $L$ is given in Appendix C. Equation (8) states that helicity is injected when the current ramps up (much like energy storage in an inductor). In past experiments, the axisymmetric period is brief due to the rapid onset of a kink which has a time-varying inductance until saturation (making it a type I process). However, under certain conditions a fixed geometry is produced, discussed further in the Results.

\section{RESULTS}

Several experimental results are categorized here according to the processes that give rise to inductive impedance: either type I ( $\dot{L}$ term dominates) or type II ( $\dot{I}$ term dominates).

\section{A. Type I process: Helicity injection with current sheets}

By carefully programming the bank to deliver just sufficient current to meet the ejection threshold it is possible to push out a single symmetric current sheet from the gun. Figure 4 shows the evolution of this current sheet in schematic and by use of a sequence of camera images obtained at the midplane of the spheromak. As the current sheet is expelled it grows asymmetries (of scale length, $l \ll R$ ) and has the appearance of a plasma "bubble." The propagation time of the current sheet out of the gun is typically Alfvénic ( $\sim$ few microseconds). Also shown in Fig. 4 are snapshots of the evolution of poloidal flux contour evolution from twodimensional simulations in SSPX geometry produced by NIMROD simulations, reproducing the phenomenon.

Figure 5 shows a typical time history of the gun current, voltage, and magnetic field at the midplane of the flux conserver as the bubble is pushed out of the gun. Initially the 

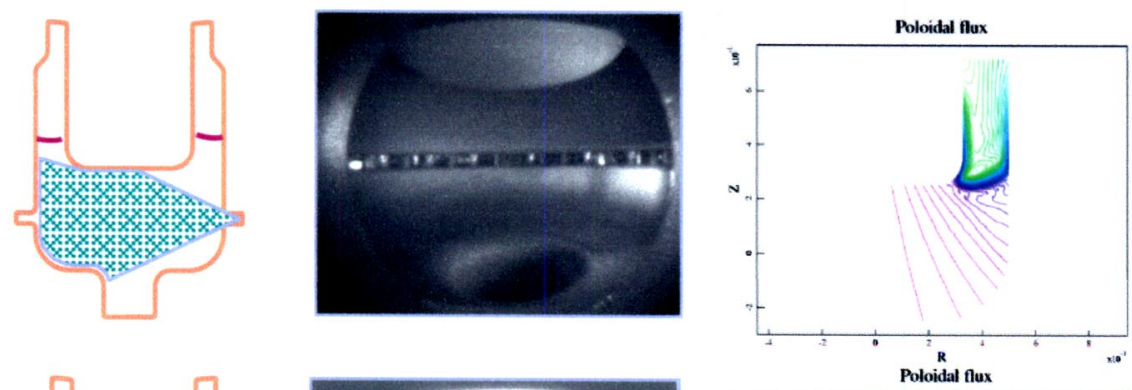

$\mathrm{t}=4.84 \times 10^{-3}$
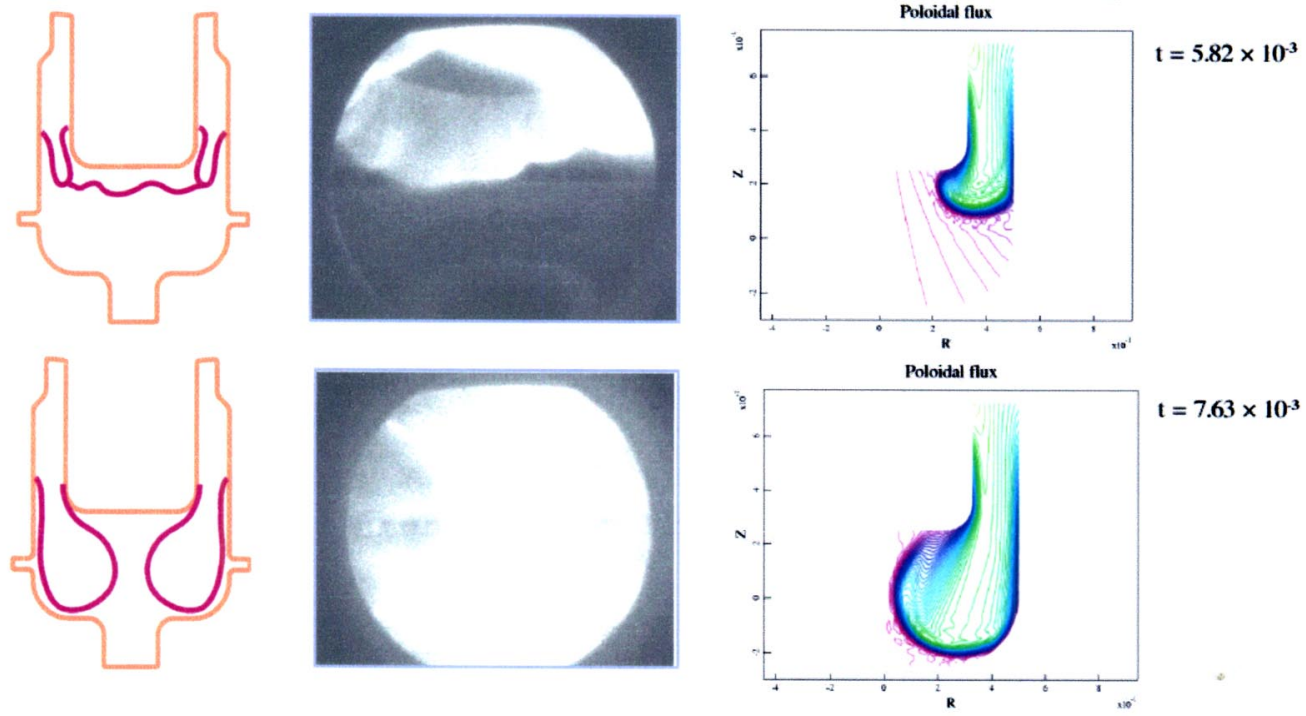

FIG. 4. Illustration with fast camera images (courtesy of CALTECH) of the ballooning of a current sheet from the gun.

voltage is determined by a fixed inductance and time-varying current $\left(V_{\text {gun }}=L \dot{I}_{\text {gun }}\right)$ as current flows in the gun, but when $I_{\text {gun }}=I_{\text {thresh }}$, the sheet expands from the gun and as it grows in size, $L$ grows to produce a $1600 \mathrm{~V}$ voltage spike. As the

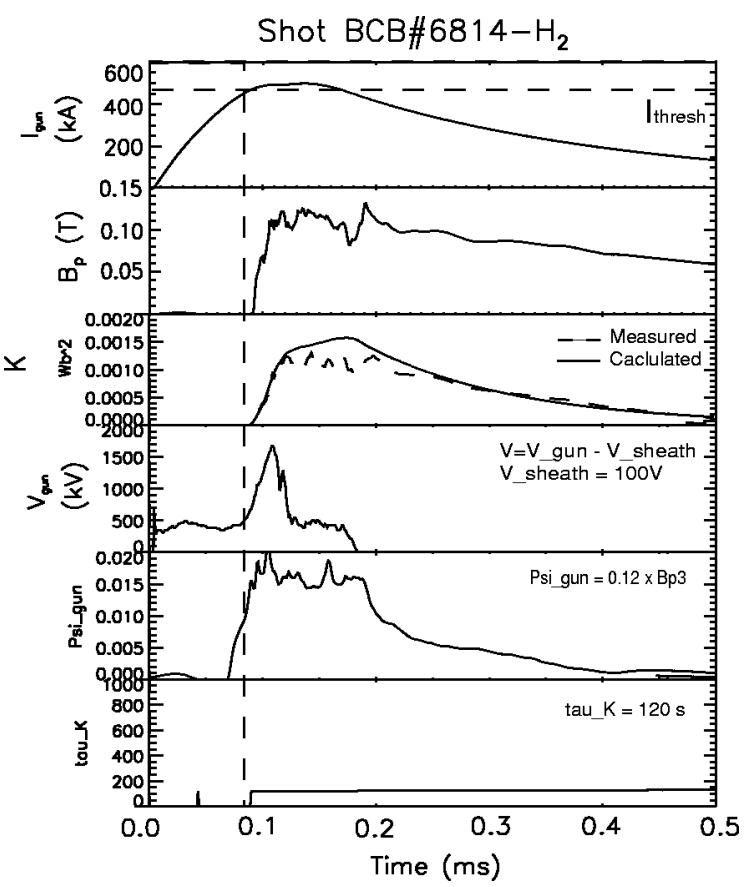

FIG. 5. Time histories of (a) gun current, (b) spheromak poloidal magnetic field strength at the midplane, (c) helicity content, (d) gun voltage, (e) injected flux, and (f) helicity decay time. current falls below the ejection threshold, the spheromak disconnects and the voltage falls to $\sim 50 \mathrm{~V}$ (consistent with a short near the breech of the gun), after which the spheromak decays. By using probes mounted at the edge in the gun, the magnetic flux expelled from the gun is observed to increase with time up to $15 \mathrm{~mW}$, then falls quickly to zero in the gap. By integrating the measured products of voltage and flux, it is possible to show the helicity evolution [Fig. 5(c)]. In this particular example, the helicity content of the spheromak is accurately predicted by knowledge of the threshold current alone: for shot 6814 where $I_{\text {thresh }}=410 \mathrm{kA}, K=1.5 e-3 \mathrm{~Wb}^{2}$. An edge magnetic coil, calibrated with CORSICA, gives the helicity of the spheromak to be $1 \times 10^{-3} \mathrm{~Wb}^{2}$. Note that the measured and predicted helicity contents of the spheromak differ: after the current sheet has been expelled from the gun, voltage falls to $\sim 300 \mathrm{~V}$ until $I_{\text {gun }}=I_{\text {thresh }}$. The measured helicity does not continue to increase after the initial voltage spike, while the integral of $2 V_{\text {gun }} \psi_{\text {gun }}$ shows a continued increase.

\section{B. Type I process: Helicity injection by multiple current filaments}

Figure 6 shows the current, voltage, and magnetic field time histories for a new operating mode (first reported in Ref. 16) that gives a continued increase of magnetic field strength until the bank runs out of charge (shot No. 7226). By maintaining $I_{\text {gun }} \sim I_{\text {thresh }}$, spheromak magnetic field strength grows monotonically and $1.5 \mathrm{kV}$ voltage spikes occur with a typical period of $10 \mu \mathrm{s}$. The voltage spikes raise 


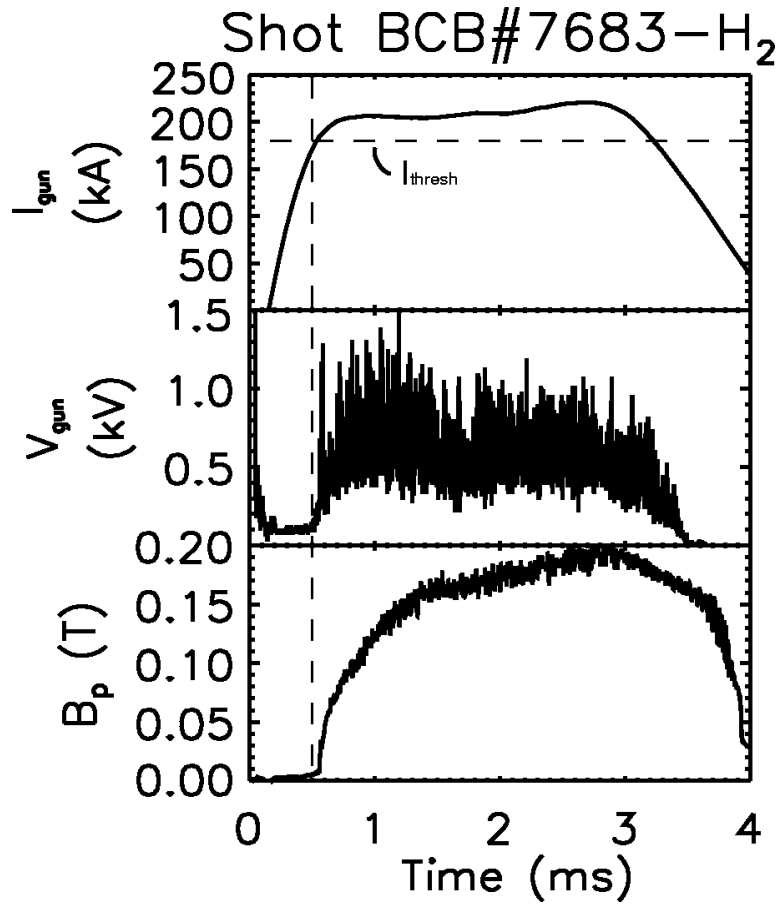

FIG. 6. Steadily building discharge.

the mean voltage by more than a factor of 2 compared with other operating modes, giving the highest sustained rate of injection for SSPX.

The origin of the voltage spikes is explored by use of a number of signal processing techniques. Figure 7 shows the auto spectra for the filtered voltage and field fluctuations, their cross-power spectrum and their coherence. The voltage fluctuations have a much broader spectrum than the magnetic field fluctuations and extend beyond $100 \mathrm{kHz}$. For this analysis, those signals above $100 \mathrm{kHz}$ are filtered out as they contribute only fractionally to the total signal. Still, it is significant that the energy in the voltage fluctuations is finite out to and beyond $100 \mathrm{kHz}$, whereas in the magnetic signals,
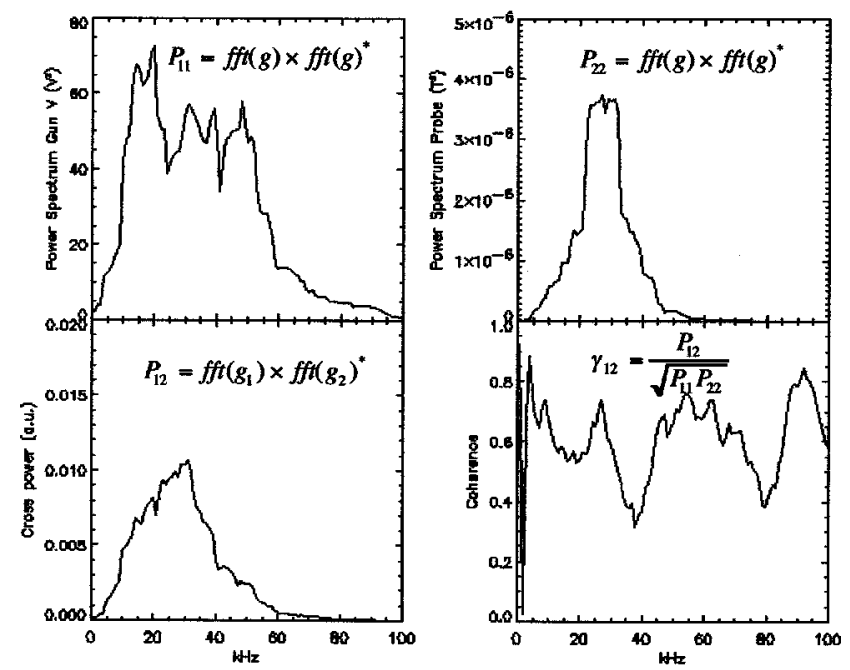

FIG. 7. Auto power spectra from the filtered gun and field fluctuations, cross spectrum, and coherence.

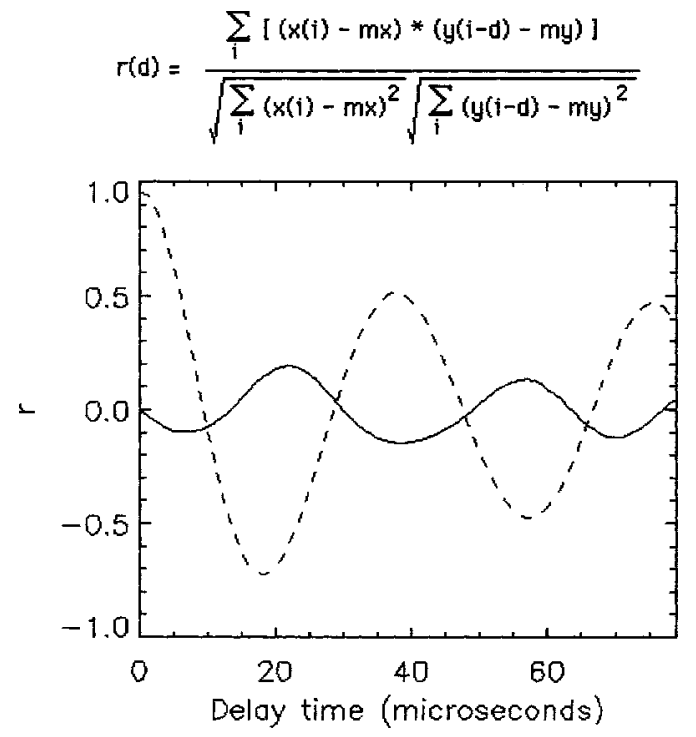

FIG. 8. Correlation of magnetic field with gun voltage (solid line) and of two magnetic field coils mounted on the insertable probes, separated by 10 $\mathrm{cm}$ vertically (dashed line). If $r$ is less than 0.5 , the signals are said to be weakly correlated.

there are virtually no signals with frequency above $50 \mathrm{kHz}$. Further, the voltage fluctuations have large amplitude from low frequency up until $60 \mathrm{kHz}$ : more than double the $n=1$ mode frequency indicating that the rotation of the doughhook (that generates the $n=1$ mode signal) is not by itself responsible for voltage fluctuations (voltage fluctuation amplitudes are at minimum at the $n=1$ mode frequency of 25 $\mathrm{kHz}$ ). This is shown further by a low cross power at all frequencies other than the $n=1$ mode frequency, and the signals are coherent $(\gamma>0.6)$ only at the mode frequency (above $50 \mathrm{kHz}$, coherence becomes meaningless as the magnetic signals fall to insignificant amplitude). The weak coherence is manifest also in the time-delay correlation. Figure 8 shows the correlation function for voltage and magnetic field fluctuations in the gun (between two coils mounted $10 \mathrm{~cm}$ apart vertically): the magnetic field appears to be well correlated in time with $r \sim 1$ falling to 0.5 over 40 or so microseconds. There is not a strong time correlation between the magnetic field and voltage: $r \sim 0.2$, but the correlation is finite due to the $n=1$. In summary, voltage fluctuations at the $n=1$ mode frequency appear to be coherent with those in the magnetic field spectrum, however these frequencies represent only a small range in frequency over which the energy of the voltage fluctuations is distributed (extending up to and beyond $100 \mathrm{kHz}$ ) for which signals are incoherent and poorly time correlated with magnetic field fluctuations.

Certain shots operated with slightly higher gun current show a change of current path and a cessation of magnetic field increase earlier in the time history. Figure 9 shows gun voltage, current, magnetic field, density, and fluctuation amplitudes from one of these discharges: note that the magnetic field rolls over at about $2 \mathrm{~ms}$, and that the gun voltage changes character from spikey to nearly constant (at $\sim 200 \mathrm{~V}$ ). What makes these discharges different is that the edge toroidal field continues to increase throughout the shot 


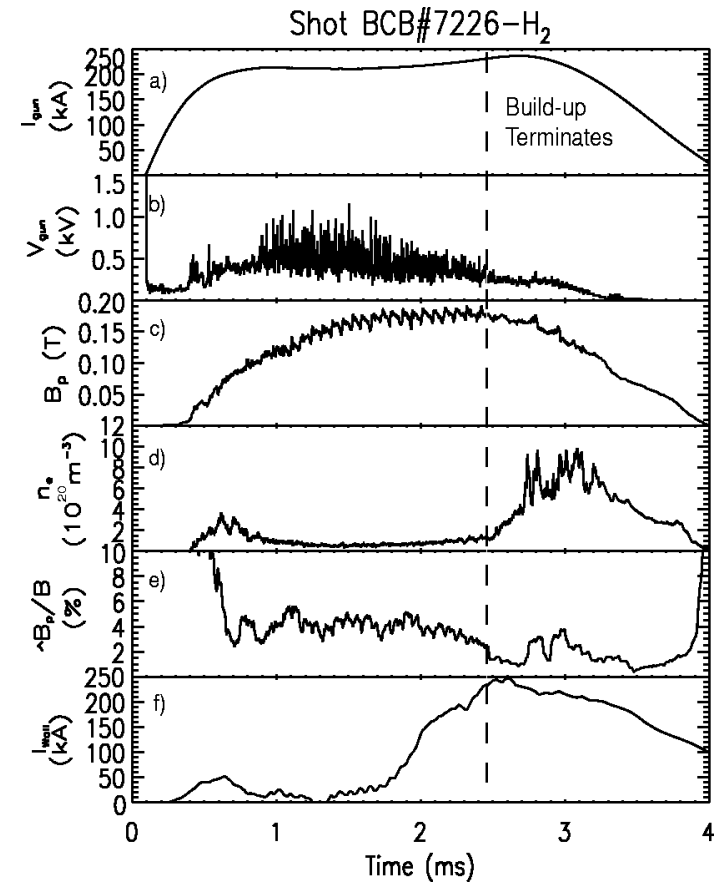

FIG. 9. A steadily building shot that ceased to build part-way through the shot.

[Fig. 9(f)] indicating a change of current path: current flows increasingly into the flux conserver instead of returning to the gun.

With both the signal analysis and knowledge of the current path, we can build a heuristic model for the origin of the voltage fluctuations (shown in Fig. 10). It is the interaction of the out-going and in-bound current paths that leads to discrete reconnection events in the "nearly stuffed" gun. A mechanism that would entail these properties would be the expulsion of multiple filaments: the helicity that is carried by this filament is additive to the spheromak helicity by further reconnection processes, and so the spheromak field strength grows with time. Further evidence for filament formation in SSPX was presented by Ryutov, ${ }^{26}$ evidenced by electrode tracks and argued on the basis of an instability arising from the temperature and energy dependences of the particle recycling coefficient.

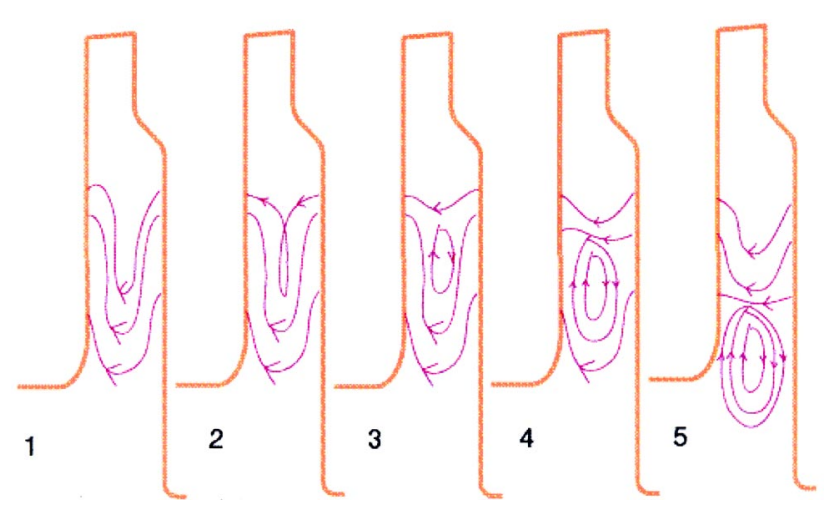

FIG. 10. Schematic origin of the gun-voltage fluctuations.

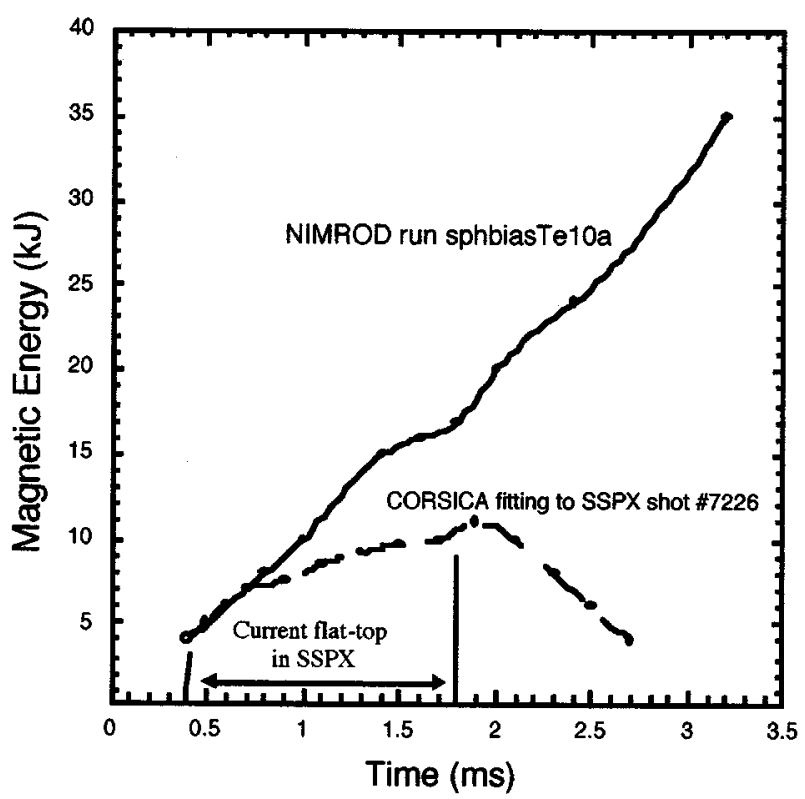

FIG. 11. NIMROD simulation shows continued magnetic energy buildup with a longer pulse.

While the voltage is set as a boundary condition in the NIMROD simulations discussed in this work, it is still possible to simulate the effect of applying a high time-average voltage for an extended period (where in the experiment it is the voltage spikes that raise the average voltage). Simulations of shot No. 7226 have qualitative and quantitative agreement. With Spitzer temperature-dependent resistivity at finite pressure (peak $\beta_{e} \sim 6 \%$ ) and constant thermal conductivities (isotropic $\chi=100 \mathrm{~m}^{2} / \mathrm{s}$ ), a continued buildup of magnetic energy is observed for pulse times of increasing length but shorter than the resistive decay time (Fig. 11). The initial increase of magnetic energy in the simulation tracks No. 7226 is fairly good. As the plasma heats, the resistivity decreases and the resistive decay time increases, which allows continued increase of the spheromak magnetic fields if the gun voltage is maintained.

\section{Type I process: Helicity injection by kink formation}

Usually in formation shots, there is an initial axisymmetric ballooning of the injector flux which injects helicity (and hence magnetic energy) into the flux conserver. If the gun current is programed to exceed the ejection threshold then an asymmetry soon develops as a MHD kink forms on the geometric axis. This transition is seen in SSPX in most shots and is characterized in the mode analysis shown in Fig. 12. The frequency of the $n=1$ mode is typically around $10-20 \mathrm{kHz}$. Determining the coherence at the $n=1$ frequency, it is apparent that the mode is globally coherent: the coherence is around unity everywhere. The discrete Fourier analysis reveals a strong $n=2$ component during the period that the $n$ $=1$ is present. This is interpreted as a harmonic distortion rather than a true $n=2$ kink of the toroidal flux. The reason is that the $n=2$ phase change occurs at twice the rate observed for the $n=1$. 

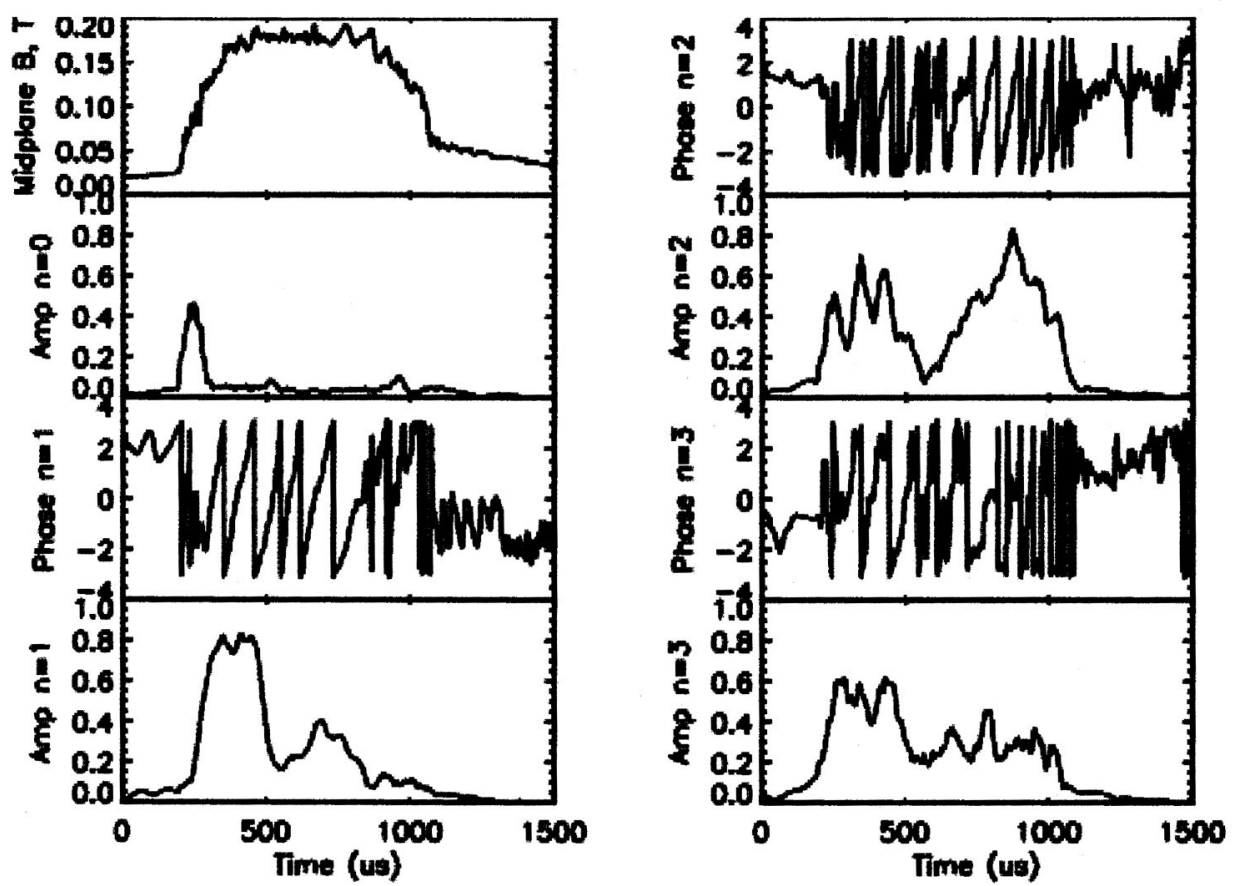

FIG. 12. Mode analysis for formation shot No. 3099. Note the initial $n=0$ mode, followed by the $n=1$ mode (and various harmonics).

In NIMROD simulations large $n=1$ MHD perturbations are observed when the plasma is strongly driven by the electrostatic field, shown in Fig. 13. The poloidal magnetic field perturbation at the outboard midplane is $\delta B_{z}(n=1) / B_{z}$ $\sim 10 \%$; however, the $n=1$ relative perturbation amplitude is $\sim 25 \%$ averaged over the volume in the simulation. Although the toroidally averaged fields in the NIMROD simulation indicate that a mean-field spheromak has formed with flux amplification similar to SSPX shots, the Poincaré surface-ofsection plots for tracing the magnetic field lines indicate short, open field lines until the driving voltage has been crowbarred (shorted out), which allows the symmetrybreaking perturbations to resistively decay and closed flux surfaces can then emerge. ${ }^{11,23}$
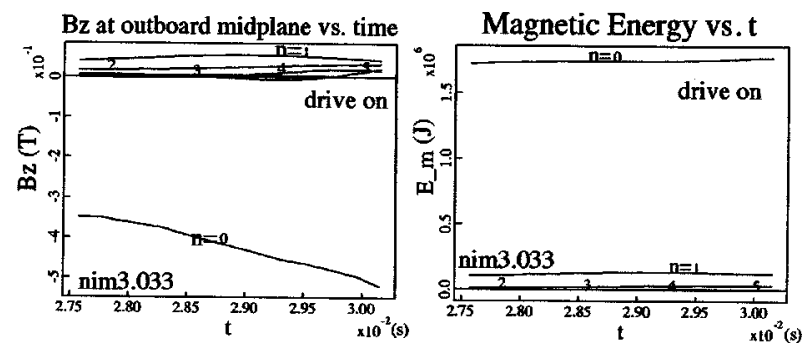

FIG. 13. (a) Vertical magnetic field Fourier analyzed in angle measured just inside the major radius of the spheromak at the vertical midplane for a simple cylinder simulation as a function of time. (b) Magnetic energy integrated over volume Fourier analyzed in angle as a function of time. Simulation parameters: bicubic finite elements $(24 \times 24)$, six toroidal Fourier modes, $\quad E_{0}=100 \mathrm{~V} / \mathrm{m}, \eta / \mu_{0}=1 / 2, \tau_{\mathrm{A}} \sim 10^{-5} \mathrm{~s}, \tau_{\mathrm{r}} \sim 8 \times 10^{-2} \mathrm{~s}, n$ $=10^{21} \mathrm{~m}^{-3}, m_{\text {ion }}=10^{3} m_{\mathrm{h}}, \chi_{\|}=10^{3} \mathrm{~m}^{2} / \mathrm{s}, \chi_{\perp}=10 \mathrm{~m}^{2} / \mathrm{s}, v_{\mathrm{A}}=2 \times 10^{6} \mathrm{~m} / \mathrm{s}$.

\section{Type II Process: Helicity injection by pulsing current along a fixed kink}

Usually, the vacuum magnetic field is programed to be radial across the mouth of the gun which can inhibit ejection. The $I d L / d t$ term, can be minimized by programing a predominately vertical vacuum field, as shown in Fig. 1, and is much like that used in the NIMROD simulations. By pulsing the gun current twice along a predominately constant inductance path the magnetic energy increases in a step-wise manner.

Two $450 \mathrm{kA}$ current pulses can be produced as shown in Fig. 14(a) by firing each half of the formation bank separately, at $1 \mathrm{~ms}$ after the sustainment pulse-forming network has fired. Also shown in Fig. 14 are the following: (b) resulting gun voltage (peaked twice at around $2 \mathrm{kV}$ ); (c) total magnetic field energy as inferred by a magnetic field coil at the midplane calibrated with CORSICA; and (d) plasma lineaveraged density measured on a chord through the magnetic axis. Close timing of the two pulses gives an increase of the stored magnetic energy from 18 to $26 \mathrm{~kJ}$, after which time, the spheromak decays. In this way, the highest magnetic fields yet observed in SSPX are produced $(0.35 \mathrm{~T}$ at the wall and $0.7 \mathrm{~T}$ at the geometric axis) and the ratio of the edge field to the injected current is higher by $25 \%$ than the previous scaling. CORSICA infers that the increase of the magnetic energy is attributable to an increase of the total current enclosed by the separatrix, i.e., pulsing increases the fraction of current flowing on closed field lines from $210 \mathrm{kA}$ to $350 \mathrm{kA}$ (an increase of $\sim 70 \%$ ). During the second pulse, the density is temporarily increased by a factor of 2 , however, it falls rapidly to a level that is consistent with most discharges 


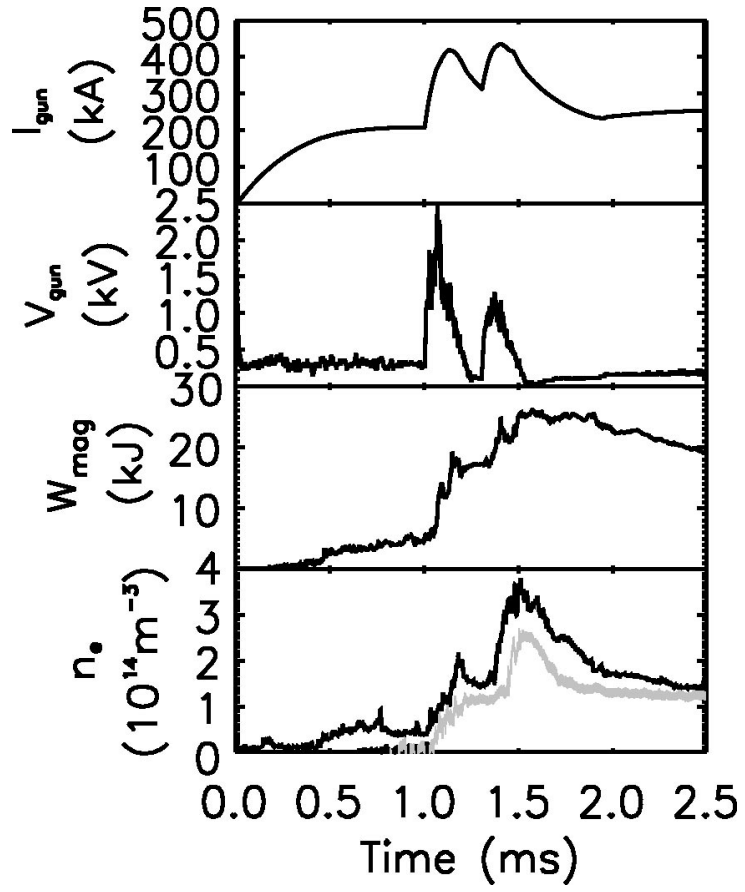

FIG. 14. Results of double pulsed operation with the $n=1$ mode.

formed with $450 \mathrm{kA}$. The gray line on Fig. 14(d) shows the attained density after around 100 shots (with intermittent $T_{i}$ gettering), and by marginally increasing the programed vacuum field. It is lower compared with the original shots indicating that the large increase of density during the second pulse can be attributed to poor surface conditioning, and the introduction of dense edge plasma. However, irrespective of surface conditioning the density collapses after the second pulse to a similar level obtained on the first pulse.

Figure 15 shows a plot of the gun voltage against the rate of change of injected current for a typical pulsed shot in order to demonstrate the phase relationship between voltage and current for these discharges. The process is identified as inductive by the linear proportionality between $V$ and $d I / d t$. Note that the absolute magnitudes differ: the first pulse has an inductance of $0.27 \mu \mathrm{H}$ and the second has $L \sim 0.2 \mu \mathrm{H}$-this is discussed below (and also in Ref. 25). There is also evidence for the buildup of magnetic energy along the geometric axis during the second pulse that is suddenly redistributed in a single event (paper submitted to PoP).

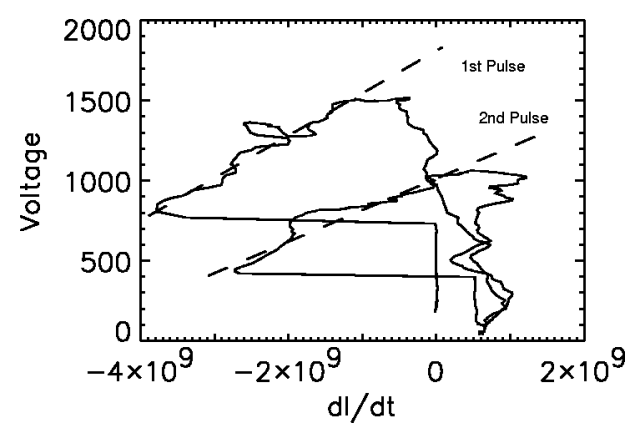

FIG. 15. $V$ vs $d I / d t$ for pulsed buildup for shot 11170 .

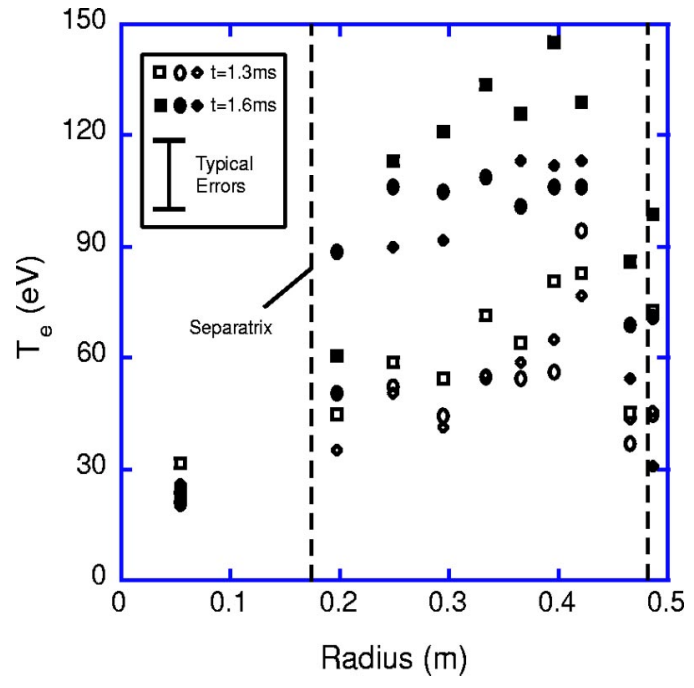

FIG. 16. Temperature profiles for pulse buildup.

While the $n=1$ is the dominant mode during the second pulse, the spectrum is quite broad: similar amplitudes out to $n=5$ indicate a strongly driven configuration, and fluctuation amplitudes are comparable to more usual modes of operating. There is no clear indication that current sheets are expelled from the gun, instead probes inserted across the mouth of the gun show a very strong asymmetry $(100 \%$ variations of poloidal and toroidal field there). This is symptomatic of the rotation of the current column that gives rise to the $n$ $=1$ mode. The asymmetry is not observed at the midplane of the spheromak, where calibration errors can explain a 5\% systematic asymmetry in the poloidal fields.

However, asymmetry is manifest quite strongly at the divertor and lower flux conserver indicating that the injected current path extends from the gun to the divertor. This path is substantiated by the observation of high wall currents (high edge toroidal field), and by camera images that reveal bright asymmetric patches. Camera images do not reveal a large kink at the geometric axis (like those observed by Lindberg), presumably because the impurities burn through to higher ionized states and do not radiate in the visible.

Typically, the temperature profile during the decay of the second pulse is peaked at the magnetic axis at around 120 $\mathrm{eV}$. Figure 16 shows a typical temperature profile (at $1.6 \mathrm{~ms}$ ), just after the second pulse. PTS was scanned throughout the time history and shows the highest temperatures at around 2 $\mathrm{ms}$. At $1.3 \mathrm{~ms}$, just before the second pulse, the average core temperature from a number of shots is $\sim 80 \mathrm{eV}$. The local electron $\beta$ is typically higher after the second pulse and reaches $\sim 6 \%$ in the core.

Increasing the time between pulses results in a quasisteady sustainment of the configuration. Figure 17 shows the time histories of the current, voltage, magnetic field, and average core electron temperature obtained from 15 similar shots. While the spheromak fields remain finite, the temperature is caused to fall during the pulses due to a large influx of cold plasma, giving also a high density for the second pulse. However, measurement of the $\beta$ profile before and after sec- 


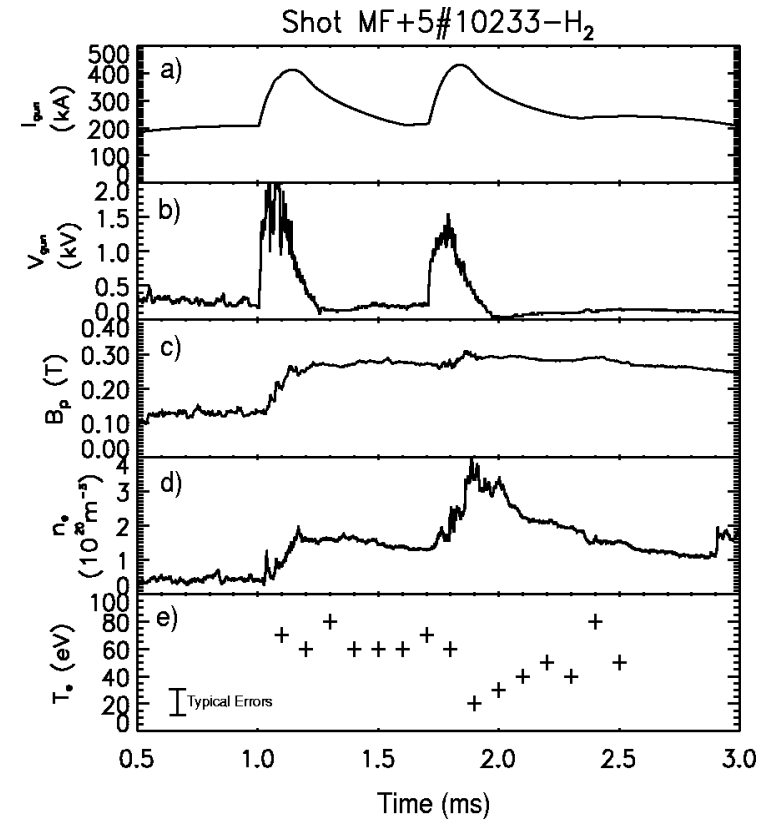

FIG. 17. From shot MF+5 No. 10223: (a) gun current; (b) gun voltage; (c) edge poloidal field at the midplane; (d) density from chord through the magnetic axis; and (e) average core temperature from a series of nominally identical shots.

ond pulse indicates that confinement recovers after the collapse with the radial profile and magnitude of $\beta$ returning to the prepulse level.

We used NIMROD to simulate the effects of driving spheromak formation with two voltage pulses. The first voltage pulse forms the spheromak, which is then allowed to decay by crowbarring the driving potential. Closed field lines emerge when the symmetry-breaking perturbations are less than a few percent in volume-averaged magnetic energy. The second pulse builds the magnetic energy to a higher level, and there is evidence for a fourfold increase in the volume of closed flux from the first to the second pulse and an increase in the total flux amplification from 4.7 to 5.1 at the times when the driving voltages were crowbarred and from 4.0 to 4.45 in decay when the good flux surfaces shown in Fig. 18 occurred. These increases are not attributable to the decrease in the total dissipation (as the resistivity is not temperature dependent in this simulation). The driving time for each pulse was $31 \mathrm{~ms}$, and the vertical electric fields near the outer radial boundary of the pillbox were $100 \mathrm{~V} / \mathrm{m}$ in both pulses. The mass density in this simulation is $\sim 10^{4}$ greater in the simulation than experiment; both velocities and times scale with the Alfvén speed so this corresponds to $\sim 0.3 \mathrm{~ms}$ in the experiment. Higher temperatures were achieved after the second pulse than after the first pulse $(95 \mathrm{eV}$ at $0.14 \mathrm{~s}$ vs $78 \mathrm{eV}$ at $0.056 \mathrm{~s}$ ). The peak plasma electron $\beta$ in the simulation was $\approx 4 \%$ at $0.14 \mathrm{~s}$, equivalent to $1.4 \mathrm{~ms}$ in the experiment.

\section{DISCUSSION}

Four operating modes have been presented alongside resistive MHD simulations showing qualitative and quantitative agreement. The essence of these operating modes was presented in Sec. II, namely, that the processes that most

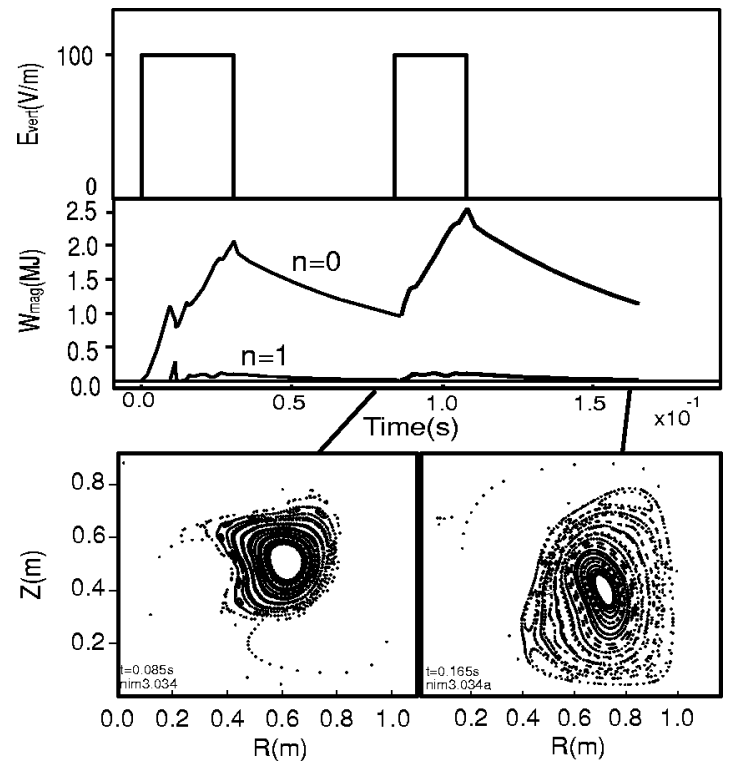

FIG. 18. NIMROD simulations of double pulsed operations.

usefully transfer helicity from the gun to the spheromak are inductive, can be expressed in simple analytic form, and can be harnessed by carefully programing the bank. We find that two new operating modes give a more favorable scaling of the magnetic field with the injected current. These new scalings are shown in Fig. 19. We discuss each of the features of these operating modes as follows.

\section{A. Inductive origin of helicity injection and the role of reconnection}

There are several more features of Eq. (7) that warrant consideration: the rate of helicity injection depends on how quickly one is able to push out a current sheet, and it is

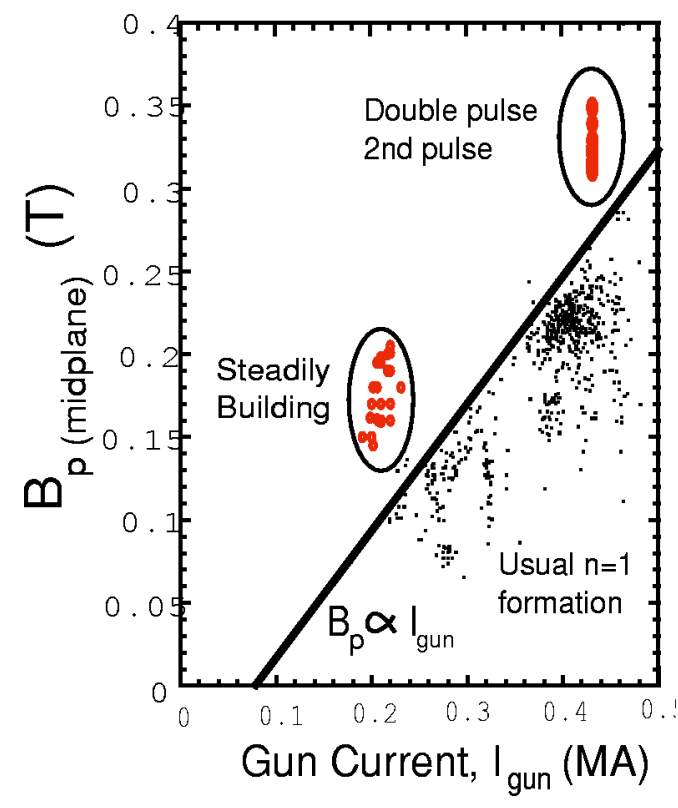

FIG. 19. Scaling of $B$ vs $I$ for operation with $n=1$ mode (solid line), operation at the ejection threshold (empty circles), and double pulsed operation (solid circles). 
possible to imagine the production of multiple current sheets. Considering the rate of injection: a less massive sheet would eject more quickly given the same forcing term, and hence one might expect there to be a sensitivity of the injection rate with gas species. This sensitivity has been observed in SSPX, particularly in shots for which there is a high pressure prefill. The production of multiple current sheets has also been observed to occur spontaneously in the experiment, and some attempts have been made to control the ejection. ${ }^{27}$ However, magnetic reconnection is necessary in order for multiple current sheets to be expelled.

The rate of change of inductance can be expressed as in terms of the ultimate inductance of the loop and a time $\tau$ : for the single current sheet, $\tau$ is the time taken for the plasmoid to fully disconnect from the gun. This description requires reconnection: it follows that if the plasmoid does not disconnect, so that another current sheet can form, the helicity of the system is defined explicitly in terms of the inductance (defined by geometry) and the current, for which the spheromak helicity will remain in proportion to $I_{g u n}$. This proportionality is observed in the experiment for many modes of operating as presented in Fig. 19 and so the linear scaling can be understood as the growth to maximum inductance of an ultimately fixed inductance current path, without reconnection. (The pressing question for such a geometry-defined helicity is the following: what limits the minimum radius of the column? An arbitrarily small column would give an arbitrarily large helicity content, for example.)

Helicity should ramp down as per Eq. (8), but this is not observed. There are no negative voltage spikes observed in the data. This can be explained somewhat trivially: the flux that is injected remains trapped in the flux conserver and cannot escape, and certainly if it did shift vertically into the gun then it would generate a negative voltage spike. Such vertical instabilities have been observed in SSPX under some unique conditions, but are not evident here.

The steadily building discharges could be described in terms of a "Roman Candle" effect whereby a current filament initiates at some point in the gun and is absorbed by the spheromak at the $x$ point near to the mouth of the gun. The precise nature of this process remains to be determined, but would have ramifications for galactic magnetic fields, which still require mechanisms, perhaps of this nature, to generate large flux amplifications.

\section{B. Scaling of the results to more interesting regimes}

Consider first the operating mode in which $I_{\text {gun }} \sim I_{\text {thresh }}$ for extended periods. As time goes on with the voltage drive fixed in NIMROD the magnetic energy in the simulation surpasses the experiment. The results shown in Fig. 11 suggest that extending the pulse coming from the driving capacitor bank can be beneficial in the experiment.

If the bank could be programed to deliver a train of pulses, how strong could the magnetic field become? The limiting spheromak magnetic field from multipulse injection is determined from the pulse repetition rate $1 / T$ and the spheromak decay rate $\tau_{K}$, set by resistive losses. Using the helicity balance equation, $d K / d t=2 \psi_{g} V_{g}-K / \tau_{K}$, the limiting helicity content can be found. Defining the helicity input from each pulse $\Delta K_{g}=\int_{t}^{t+\delta t} 2 \psi_{g} V_{g} d t$, where $\delta t$ is the pulse width of each pulse in the pulse train, and assuming constant $\tau_{K}$, the limiting helicity content is given by $K_{\infty}=\Delta K_{g}\{1$ $\left.-\exp \left(-T / \tau_{K}\right)\right\}^{-1}$. This relation balances the input rate from each pulse with the helicity decay between pulses $\Delta K_{g}\{1$ $\left.-\exp \left(-T / \tau_{K}\right)\right\}$ and is for the helicity at the end of each pulse. In this case (with $T=300 \mu \mathrm{s}$ and $\tau_{K}=1000 \mu \mathrm{s}$ ), one would expect $K_{\infty}=3.8 \Delta K_{g}$, providing of course that the spheromak dissipation time does not grow with time, in which case there may not actually be a limit to the buildup of helicity. ${ }^{28}$ Perhaps surprisingly, we see a doubling of core temperature from the first to the second pulse, which is evidence in favor of such an optimistic projection. However, we are assuming also that $\Delta K_{g}$ does not change on each pulse (which seems not to be the case here: $V_{\text {gun }}$ is lower on the second pulse by almost a factor of 2). Thus, a natural experiment is to add third and even fourth pulses, and monitor the temperature. This is unfortunately not presently possible in the experiment.

Given observed scalings, a simple model has been generated to determine the requirements to reach higher temperatures. The equations used in this model are to be found in Appendix A. Three scalings based on observation are used: (1) the scaling of temperature with density and field at approximately constant $\beta ;{ }^{15}$ (2) the scaling of density with gun current, in approximately linear form; and (3) the new scalings for the magnetic field with gun current presented here. A Bessel function model is used to infer internal field and current profiles, scaled to a measured edge magnetic field to calculate $J$ in the core and hence the Ohmic heating power and a heating time. This heating time determines the minimum time constraint on the sustainment bank. Hence the size of the sustainment bank can be determined, given also an observed coupling efficiency. We can also calculate the electrode heating, given calorimetry performed on the inner electrode. Given constraints on electrode heating (due to surface melting), and on bank size (due to cost), we are able to determine which operating mode is the most favorable to explore in the future.

As an example of the model, consider the usual SSPX discharges for which we have observed $200 \mathrm{eV}$ with $400 \mathrm{kA}$ on the formation bank and $200 \mathrm{kA}$ on the sustainment. The core temperature is $200 \mathrm{eV}$, edge field is $0.26 \mathrm{~T}$, core density is $7 e-19 \mathrm{~m}^{-3}$, total magnetic energy is $22 \mathrm{~kJ}$ (in agreement with CORSICA), the coupled formation bank energy needed to achieve this is $150 \mathrm{~kJ}$, the ohmic heating time is $0.3 \mathrm{~ms}$, and would require a sustainment bank of only $400 \mathrm{~kJ}$ to maintain stability until $200 \mathrm{eV}$ is reached. The simple scaling model shows that for pulsed operation, it would be possible to produce a $1 \mathrm{keV}$ spheromak by producing two pulses of 1 MA. An intermediate step to attain this would be to install a modular bank that would pulse two pulses of $600 \mathrm{kA}$ (or a train of lower current pulses at $400 \mathrm{kA}$ ). Such a scenario would allow the production of a $0.5 \mathrm{~T}, 500 \mathrm{eV}$ spheromak. Such a step would be necessary for further testing of both the scaling model and the NIMROD simulations. 


\section{CONCLUSION}

We have demonstrated two new operating modes in SSPX that raise the scaling of the field to injected current significantly above what has been obtained previously. We understand the helicity-injection processes that cause this increase in scaling to result from inductive processes in the plasma. Comparison with NIMROD simulations, operated in SSPX-similar parameter space, shows similar features for all of the experimental operating modes. Using a simple scaling model and the understanding gained from scalings observed in the experiment, we are able to predict what bank modifications need to be made in order to produce a spheromak of a given temperature. Further, we indicate that a bank modification should give rise to $500 \mathrm{eV}$ spheromaks. This modification is therefore a necessary step towards the production of high temperature plasmas, which would give additional scaling information and would serve as a critical test for NIMROD code.

\section{ACKNOWLEDGMENTS}

The authors would like to acknowledge useful discussions with Dmitri Ryutov and Ken Fowler, and other SSPX team members for keeping the machine operating. An earlier version of this paper was presented as an invited talk at the American Physical Society Division of Plasma Physics meeting, November, 2003. This work was performed under the auspices of the U.S. Department of Energy by the University of California Lawrence Livermore National Lab under Contract No. W-7405-ENG-48.

\section{APPENDIX A: SCALING MODEL}

The scaling model entails the following linear scaling relations observed experimentally:

$$
\begin{aligned}
& T_{e}(\mathrm{eV})=\frac{\beta(\%) B_{p}^{2}(\mathrm{~T})}{2 \mu_{0} n_{e}\left(\mathrm{~m}^{-3}\right)}, \\
& B_{p}(\mathrm{~T})=c_{1} I_{\text {gun }}(\mathrm{A}), \\
& n_{e}\left(\mathrm{~m}^{-3}\right)=c_{2} I_{\text {gun }}(\mathrm{A}), \\
& W_{B}(\mathrm{~J})=\frac{B_{p}^{2}(\mathrm{~T})}{2 \mu_{0}} \Delta V\left(\mathrm{~m}^{3}\right), \\
& E_{\text {bank }}(\mathrm{J})=W_{B}(\mathrm{~J}) / f(\%),
\end{aligned}
$$

where $f$ is the empirically derived efficiency of formation, which is typically small $(<10 \%)$. The time taken for the plasma to heat to a given temperature is determined by the Ohmic heating power calculated by a Bessel function approximation for the magnetic fields scaled in proportion to the edge poloidal field $B_{p}$, and a Spitzer resistivity:

$$
t_{\text {heat }}(\mathrm{s})=\frac{k}{c_{3}} \frac{2}{5} T^{5 / 2}(\mathrm{eV}),
$$

where $k=e n$ and $c_{3}=5.2 \times 10^{-5} Z_{e f f}|\lambda| J^{2} \Delta V$.

\section{APPENDIX B: SHARP BOUNDARY MODEL}

Here we use a simple slab model to illustrate the ejection threshold. Toroidal flux is generated by axial current flow in the inner electrode and poloidal flux is generated by the injector solenoid. Injector gap distance is $\Delta$, and has a radius $R(\gg \Delta)$. We assume that the poloidal flux becomes compressed into a channel of width $\delta$ and length $l$. The toroidal and poloidal fluxes are then written as

$$
\begin{aligned}
& \Phi=B_{\vartheta}(\Delta-2 \delta) l, \\
& \Psi=2 \pi R B_{p} \delta .
\end{aligned}
$$

By setting the poloidal and toroidal magnetic field components to be equal (magnetic pressure equal at threshold), the magnetic energy can be expressed as

$$
W_{B}=\frac{\Psi \Phi \Delta}{2 \mu_{0} \delta(\Delta-2 \delta)},
$$

then by minimizing the magnetic energy with respect to the flux-channel width $(d \mathrm{~W} / d \delta=0)$ one obtains $\delta=\Delta / 4$. The threshold for ejection can be written simply as $\lambda_{\text {crit }}=4 / \Delta$ (where a slab model would give $\pi / \Delta$ ). For SSPX, $\lambda_{\text {crit }}$ $\sim 19.1 \mathrm{~m}^{-1}$.

\section{APPENDIX C: INDUCTANCE OF A COAXIAL SCREW PINCH}

In the axisymmetric approximation, a column has a magnetic field

$$
B=\frac{\mu_{0}}{r} \int_{0}^{r} j(r) d r .
$$

Assume that the current is returning in a cylinder at radius $r_{m}$ and length $L_{m}$. The magnetic energy is thus

$$
W=\int \frac{B^{2}}{2 \mu_{0}} d V=\pi L_{m} \mu_{0} \int_{0}^{r} \frac{d r}{r}\left[\int_{0}^{r} j r d r\right]^{2} .
$$

Suppose the column has a radius $a$, the current is $I$, and the current density is flat. Then, we can write

$$
\begin{aligned}
W & =\frac{L_{m} \mu_{0}}{4 \pi} I^{2}\left\{\int_{0}^{a} \frac{d r}{r}\left[\int_{0}^{r}\left(\frac{r}{a}\right)^{2} d r\right]^{2}\right\}, \\
B_{\text {in }} & =\frac{\mu_{0}}{r} \int_{0}^{r} J r d r=\frac{\mu_{0} I r}{2 \pi a^{2}}, \\
B_{\text {out }} & =\frac{\mu_{0} I}{2 \pi r}, \\
W & =\int \frac{B^{2}}{2 \mu_{0}} d V=\frac{\pi L_{m}}{\mu_{0}}\left[\int_{0}^{a} B_{\text {in }}^{2} r d r\right]+\left[\int_{a}^{b} B_{\text {out }}^{2} r d r\right] \\
& =\frac{L_{m}}{16 \pi} \mu_{0}[1+4 \ln (b / a)] I_{\text {gun }}^{2}
\end{aligned}
$$

and given 


$$
\begin{aligned}
& W=1 / 2 L I^{2}, \\
& L=\frac{L_{m}}{8 \pi} \mu_{0}[1+4 \ln (b / a)],
\end{aligned}
$$

which yields the inductance in terms of the ratio of flux conserver-to-column radius (where the current returns).

${ }^{1}$ T. R. Jarboe, Plasma Phys. Controlled Fusion 36, 945 (1994).

${ }^{2}$ H. Alfvén, L. Lindberg, and P. Mitlid, J. Nucl. Energy C1, 116 (1960).

${ }^{3}$ L. Lindberg and C. T. Jacobsen, Phys. Fluids, S44 (1963); Astrophys. J. 133, 1043 (1960); H. Alfvén, ibid. 133, 1049 (1960).

${ }^{4}$ W. C. Turner, G. C. Goldenbaum, E. H. A. Granneman, J. H. Hammer, C. W. Hartman, D. S. Prono, and J. Taska, Phys. Fluids 26, 1965 (1983).

${ }^{5}$ T. R. Jarboe, I. Henins, A. R. Sherwood, C. W. Barnes, and H. W. Hoida, Phys. Rev. Lett. 51, 39 (1983).

${ }^{6}$ M. Yamada, Nucl. Fusion 25, 1327 (1985).

${ }^{7}$ Y. Ono, A. Morita, M. Katsurai, and M. Yamada, Phys. Fluids B 5(10), 3691 (1993).

${ }^{8}$ M. R. Brown and P. M. Bellan, Phys. Rev. Lett. 64, 2144 (1990).

${ }^{9}$ M. Yamada, Y. Ono, A. Hayakawa, M. Katsurai, and F. W. Perkins, Phys. Rev. Lett. 65, 721 (1990).

${ }^{10}$ R. C. Duck, P. K. Browning, G. Cunningham, S. J. Gee, A. al-Karkhy, R. Martin, and M. G. Rusbridge, Plasma Phys. Controlled Fusion 39, 715 (1997).

${ }^{11}$ C. R. Sovinec, J. M. Finn, and D. del-Castillo-Negrete, Phys. Plasmas 8, 475 (2001).

${ }^{12}$ S. A. Colgate, H. Lui, and V. Pariev, Phys. Plasmas 8, 2425 (2001).

${ }^{13}$ A. al-Karkhy, P. K. Browning, G. Cunningham, S. J. Gee, and M. G. Rusbridge, Phys. Rev. Lett. 70, 1814 (1993).
${ }^{14}$ H. S. McLean, S. Woodruff, E. B. Hooper, R. H. Bulmer, D. N. Hill, C. Holcomb, J. Moller, B. W. Stallard, R. D. Wood, and Z. Wang, Phys. Rev. Lett. 88, 125004 (2002)

${ }^{15}$ B. W. Stallard, D. N. Hill, C. Holcomb, Proceedings of EPS Conference on Controlled Fusion Plasma Physics, Madeira, 2001.

${ }^{16} \mathrm{~S}$. Woodruff, D. N. Hill, B. W. Stallard, R. Bulmer, B. Cohen, C. T. Holcomb, E. B. Hooper, H. S. McLean, J. Moller, and R. D. Wood, Phys. Rev. Lett. 90, 095001 (2003).

${ }^{17}$ M. G. Rusbridge, S. J. Gee, P. K. Browning, G. Cunningham, R. C. Duck, A. al-Karkhy, R. Martin, and J. W. Bradley, Plasma Phys. Controlled Nucl. Fusion Res. 39, No. 5, 683 (1997).

${ }^{18}$ R. H. Cohen, H. L. Berk, B. I. Cohen, T. K. Fowler, E. B. Hooper, L. L. LoDestro, E. C. Morse, L. D. Pearlstein, T. D. Rognlien, D. D. Ryutov, C. R. Sovinec, and S. Woodruff, Nucl. Fusion 43, 1220 (2003).

${ }^{19}$ S. Woodruff, Bull. Am. Phys. Soc. 48, 150 (2003).

${ }^{20}$ Magnetic Helicity in Space and Laboratory Plasmas, Geophysical Monograph Vol. 111, edited by M. R. Brown, R. C. Canfield, A. A. Pevtsov (American Geophysical Union, Washington, D.C., 1999).

${ }^{21}$ T. R. Jarboe, F. J. Wysocki, J. C. Fernandez, I. Henins, and G. J. Marklin, Phys. Fluids B 2, 1342 (1990).

${ }^{22}$ The NIMROD Team, C. R. Sovinec, T. A. Gianakon, E. D. Held, S. E. Kruger, and D. D. Schnack, Phys. Plasmas 10, 1727 (2003).

${ }^{23}$ The NIMROD Team, C. R. Sovinec, A. H. Glasser, T. A. Gianakon, D. C. Barnes, R. A. Nebel, S. E. Kruger, D. D. Schnack, S. J. Plimpton, A. Tarditi, and M. Chu, J. Comput. Phys. 195, 355 (2004).

${ }^{24}$ E. B. Hooper, D. Pearlstein, and D. D. Ryutov, Nucl. Fusion 39, 863 (1999).

${ }^{25}$ B. W. Stallard et al., Phys. Plasmas 10, 2912 (2003).

${ }^{26}$ D. D. Ryutov, D. N. Hill, E. B. Hooper, Plasma Phys. Rep. 29, 605 (2003).

${ }^{27}$ S. Woodruff, Bull. Am. Phys. Soc. 47, 59 (2002).

${ }^{28}$ T. K. Fowler, Fusion Technol. 29, 206 (1996). 